\title{
Distribution of crystalloid fluid infused during onset of anesthesia-induced hypotension: a retrospective population kinetic analysis
}

Robert G. Hahn ${ }^{1,2}$ (D)

\begin{abstract}
Background: Induction of anesthesia causes a drop in arterial pressure that might change the kinetics of infused crystalloid fluid. The aim of this report is to provide a mathematical view of how fluid distributes in this setting.

Methods: Data were retrieved from three studies where 76 patients (mean age 63 years, mean body weight $66 \mathrm{~kg}$ ) had received approximately $1.1 \mathrm{~L}$ of Ringer's solution over $60 \mathrm{~min}$ by intravenous infusion before and during induction of spinal, epidural, or general anesthesia. A population kinetic model was used to analyze the fluid distribution and its relationship to individual-specific factors. Frequent measurements of blood hemoglobin and the urinary excretion served as dependent variables.

Results: Before anesthesia induction, distribution to the extravascular space was threefold faster than elimination by urinary excretion. Both distribution and elimination of infused fluid were retarded in an exponential fashion due to the anesthesia-induced decrease in the mean arterial pressure (MAP). A decrease in MAP from 110 to $60 \mathrm{mmHg}$ reduced the rate of distribution by $75 \%$ and the rate of elimination by $90 \%$. These adaptations cause most of the infused fluid to remain in the bloodstream. Age, gender, type of anesthesia, and the use of ephedrine had no statistically significant effect on plasma volume expansion, apart from their possible influence on MAP.

Conclusion: The decrease in MAP that accompanies anesthesia induction depresses the blood hemoglobin concentration by inhibiting both the distribution and elimination of infused crystalloid fluid. The report provides mathematical information about the degree of these changes.
\end{abstract}

Keywords: Anesthesia, Intravenous, Anesthesia, Regional, Hypotension, Vascular, Pharmacokinetics, Therapy, Fluid

\section{Introduction}

Crystalloid fluid is often administered intravenously (i.v.) during induction of anesthesia (McCrae and Wildsmith 1993), although this fluid is considered to have a poor plasma volume-expanding effect (Jacob et al. 2012). Volume loading before the induction (pre-loading) does not prevent a drop in arterial pressure (Coe and Revenäs,

Correspondence: robert.hahn@ki.se; r.hahn@telia.com

${ }^{1}$ Research Unit, Södertälje Hospital, 15286 Södertälje, Sweden

${ }^{2}$ Karolinska Institutet at Danderyds Hospital (KIDS), Stockholm, Sweden
1990; Ewaldsson and Hahn 2005) while providing the fluid during the onset of anesthesia (co-loading) can reduce the magnitude of the drop, which is best studied in parturients (James and Dyer 2016).

One reason for why co-loading maintains the arterial pressure better than pre-loading might be that more volume remains in the plasma in close connection with the infusion. A larger proportion of infused fluid is known to remain in the plasma when hypotension has just been induced (Hahn 1992). A recent kinetic study in which

(c) The Author(s). 2021 Open Access This article is licensed under a Creative Commons Attribution 4.0 International License, which permits use, sharing, adaptation, distribution and reproduction in any medium or format, as long as you give appropriate credit to the original author(s) and the source, provide a link to the Creative Commons licence, and indicate if changes were made. The images or other third party material in this article are included in the article's Creative Commons licence, unless indicated otherwise in a credit line to the material. If material is not included in the article's Creative Commons licence and your intended use is not permitted by statutory regulation or exceeds the permitted use, you will need to obtain permission directly from the copyright holder. To view a copy of this licence, visit http://creativecommons.org/licenses/by/4.0/. The Creative Commons Public Domain Dedication waiver (http://creativecommons.org/publicdomain/zero/1.0/) applies to the data made available in this article, unless otherwise stated in a credit line to the data. 
the fluid was given only after the induction showed an arrested distribution that resolved only $20 \mathrm{~min}$ later (Hahn and Nemme 2020), resulting in a plasma volume expansion that temporarily approached $100 \%$ of the infused amount. Besides being of physiological interest, the excessive volume expansion is mirrored by a reduction of the blood hemoglobin $(\mathrm{Hb})$ concentration that affects oxygen delivery, the planning of deliberate hemodilution, and estimates of the blood loss allowed before initiating erythrocyte transfusion.

The aim of the present report was to obtain a mathematical analysis of what governs the distribution of crystalloid fluid when given during the onset of anesthesia. The report is based on a population kinetic analysis based on plasma dilution and urinary excretion data derived from studies in which crystalloid fluid was given to clinical patients as continuous infusion before, during, and after the onset of spinal, epidural, or general anesthesia. Special consideration was given to the role of the mean arterial pressure (MAP), but other factors, such as gender and the choice of fluid, were also evaluated as having potential importance regarding this aspect of the adaptation to the anesthetized state.

\section{Methods}

This study is a retrospective population kinetic analysis that uses data from three previously published studies of fluid distribution during induction of anesthesia (Ewaldsson and Hahn 2001, 2005; Li et al. 2007). The analysis comprised pooled data from 76 infusions in which lactated or acetated Ringer's solution had been administered by i.v. infusion in a similar way to patients during induction of spinal, epidural, or general anesthesia. All three studies had been approved by the appropriate Ethics Committee before the first patient was enrolled. Written informed consent was obtained from all patients before starting the study.

\section{Anesthesia procedures}

The infusions were initiated between 7 and 9 AM. The patients had fasted overnight and were placed on a bed to rest for $30 \mathrm{~min}$ to reach a hemodynamic steady state. In the first two studies, a cannula was placed in the cubital vein of each arm, one for blood sampling and the other for infusion of fluid. The arm used for blood sampling was placed on a body-warm heating pad. In the third study, arterial blood was sampled.

Anesthesia was usually induced when $1 / 3$ of the infusions had been administered. The types of anesthesia used in the three studies are shown in Table 1. Spinal anesthesia was induced in the left lateral decubitus position using a 25G Whitacre needle. The subarachnoid space was punctured in the $\mathrm{L}_{3}$ and $\mathrm{L}_{4}$ interspace or, if that was unsuccessful, in the $\mathrm{L}_{2}-\mathrm{L}_{3}$ interspace. Plain bupivacaine (2.4-3.4 mL; Marcain-Spinal, $5 \mathrm{mg} / \mathrm{mL}$; AstraZeneca, Södertälje, Sweden) was injected as required to achieve surgical anesthesia.

Epidural anesthesia was applied through a 17-gauge Tuohy needle with the patient in the same body position. Increments of 3-5 mL 0.5\% ropivacaine (Pharmacia, AstraZeneca, Germany) were injected every $5 \mathrm{~min}$ until a surgical block was achieved.

General anesthesia was induced with propofol (1.5 $\mathrm{mg} / \mathrm{kg})$, midazolam $(0.05 \mathrm{mg} / \mathrm{kg})$, sufentanil $(0.6 \mu \mathrm{g} / \mathrm{kg})$, and rocuronium bromide $(0.6 \mathrm{mg} / \mathrm{kg})$, and anesthesia was then maintained with propofol $(3 \mathrm{mg} / \mathrm{kg} / \mathrm{h})$ and rocuronium bromide $(4 \mathrm{mg} / \mathrm{h})$.

\section{Measurements}

The plasma volume was expanded by infusing the Ringer solution i.v. using infusion pumps. During and after

Table 1 Demographic data for the cohorts used for population volume kinetic analysis

\begin{tabular}{|c|c|c|c|}
\hline & Ewaldsson and Hahn 2001 & Ewaldsson and Hahn 2005 & Li et al. 2007 \\
\hline Females/males & $1 / 4$ & $9 / 11$ & $18 / 33$ \\
\hline Infusions ( $N$ ) & 5 & 20 & 53 \\
\hline Age (years) & $70(54-80)$ & $65(33-80)$ & $61(24-86)$ \\
\hline Body weight (kg) & $85(79-106)$ & $76(54-120)$ & $61(45-96)$ \\
\hline Fluid volume (mL/kg) & 15 & 20 & $\approx 17$ \\
\hline Infusion time (min) & 40 & 60 & 60 \\
\hline Infusion rate (mL/min/kg) & 0.375 & 0.333 & 0.286 \\
\hline Regional/general anesthesia & $5 / 0$ & $9 / 11$ & $20 / 33$ \\
\hline Type of regional anesthesia & Spinal & Spinal & Epidural \\
\hline Induction at (min) & 20 & 20 & 15 \\
\hline Patients given ephedrine $(N)$ & 1 & 5 & 7 \\
\hline Study duration (min) & 50 & 60 & 60 \\
\hline
\end{tabular}

Data are the mean (range) 
these infusions, venous blood $(3-4 \mathrm{~mL})$ was withdrawn to measure the $\mathrm{Hb}$ concentration and the hematocrit (Hct) on the apparatus used for routine measurements in the hospital's Clinical Chemistry Laboratory. The samples were withdrawn every 3-5 min in a standardized manner to ensure a coefficient of variation $(\mathrm{CV})$ of about $1 \%$. The baseline sample was drawn in duplicate, and the mean of the two concentrations was used in subsequent calculations.

The excreted urine was collected via an indwelling catheter, which had been inserted into the bladder under topical anesthesia before the studies started.

MAP was measured non-invasively with an automatic device (Datex AS3, Datex, Helsinki, Finland) (Ewaldsson and Hahn 2001, 2005), while invasive measurements were performed and displayed on a multifunction Datex-Ohmeda instrument (Hoevelaken, The Netherlands) when an arterial line had been established (Li et al. 2007).

An intravenous bolus dose of $5 \mathrm{mg}$ of ephedrine, which could be repeated if necessary, was given if the systolic arterial pressure fell to $60 \%$ of baseline or if the patient experienced near-fainting symptoms (nausea, sweating, and bradycardia). No other vasopressor was used.

\section{Kinetic analysis}

Population (mixed effects) kinetics is an industrystandard tool for evaluating and recommending dosing regimens for drugs with regard to individual-specific factors, such as age, gender, and body weight (Heeremans et al. 2010). The volume kinetic method is a modification of drug pharmacokinetics for the study of infusion fluids, but it differs from conventional pharmacokinetics in that the fluid compartments have expandable walls. A benefit of this approach is that it allows analysis of dynamic events, as this is difficult to achieve with radioactive tracer methods.

Volume kinetics is based on repeated measurement of the blood $\mathrm{Hb}$ concentration, which is the inverse of the blood water concentration (Hahn 2020). Infusion fluids contain almost exclusively water; therefore, $\mathrm{Hb}$ changes are an index of the water volume that rapidly equilibrates with the circulating blood.

A two-volume kinetic model with micro-constants was simultaneously fitted to all data for the dependent variables, which were the frequently measured plasma dilution and the urinary excretion.

The appropriateness of all fixed parameters was challenged one by one to arrive at an optimal base model. Thereafter, the influence of covariates on the fixed parameters was tested sequentially, as guided by a reduction in the residual error for the model (Owen and Fiedler-Kelly 2014).

\section{Base model}

In the finally used base model, fluid is infused at rate $R_{\mathrm{o}}$ to expand the volume of a central body fluid space $V_{\mathrm{c}}$ to $v_{\mathrm{c}}$. The volume expansion is written $\left(v_{\mathrm{c}}-V_{\mathrm{c}}\right)$.

Distribution of the fluid to a peripheral body fluid space is governed by a rate constant $k_{12}$, and the flow from the central to the peripheral space at any time is given by the product of $k_{12}$ and $\left(v_{c}-V_{\mathrm{c}}\right)$. Similarly, the elimination is the product of the volume expansion of $V_{\mathrm{c}}$ at any time and an elimination rate constant, $k_{10}$ (Fig. 1A).

The differential equations are:

$$
\begin{aligned}
& \mathrm{d} v_{c} / \mathrm{dt}=R_{\mathrm{o}}-k_{12}\left(v_{c}-V_{c}\right)-k_{10}\left(v_{c}-V_{c}\right) \\
& \mathrm{d} U / d t=k_{10}\left(v_{c}-V_{c}\right)
\end{aligned}
$$

where $U$ is the measured urinary excretion.

The plasma dilution is used to indicate the volume expansion of $V_{\mathrm{c}}$ resulting from the infusion. Hence:

$$
\left(v_{c}-V_{c}\right) / V_{c}=[(\mathrm{Hb} / \mathrm{hb})-1] /(1-\text { Hematocrit })
$$

Symbols in capital letters denote baseline values. A minor correction is made for the effects of blood sampling on the plasma dilution (Hahn 2010).

\section{Covariates}

A search for trends in plots of random effects (eta:s) was used to identify covariate candidates. A trend in an eta plot could suggest the existence of a significant covariate. The most promising candidate parameters were then tested, one by one, by adding them to the base model in a diagonal design, which assumes no correlation between random effects. The threshold for accepting one curvefit as significantly better than another was guided by a reduction of $>3.8$ points in the goodness-of-fit $(-2 \mathrm{LL}=$ $\log$ likelihood) for the model; where $>6.6$ points represents $P<0.01$. The base model with the significant covariates added to it constituted the final model and had the lowest residual error of all runs.

Gender, age, body weight, general or regional anesthesia (spinal/epidural), type of Ringer's (lactated or acetated), the use of ephedrine, and the rate of infusion per kilo body weight, as well as various time factors, were sequentially tested as covariates to the three fixed parameters in the model. The mean arterial pressure (MAP) and the heart rate were evaluated as time-varying covariates, which mean that a new value was considered for each point of measurement. Both the crude value of MAP and the change in MAP from baseline were tested.

\section{Parameter estimation}

The fixed parameters in the model and the statistically significant covariates, if any, were estimated simultaneously using the Phoenix software for nonlinear mixed effects (NLME), version 1.3 (Pharsight, St. Louis, MO) 

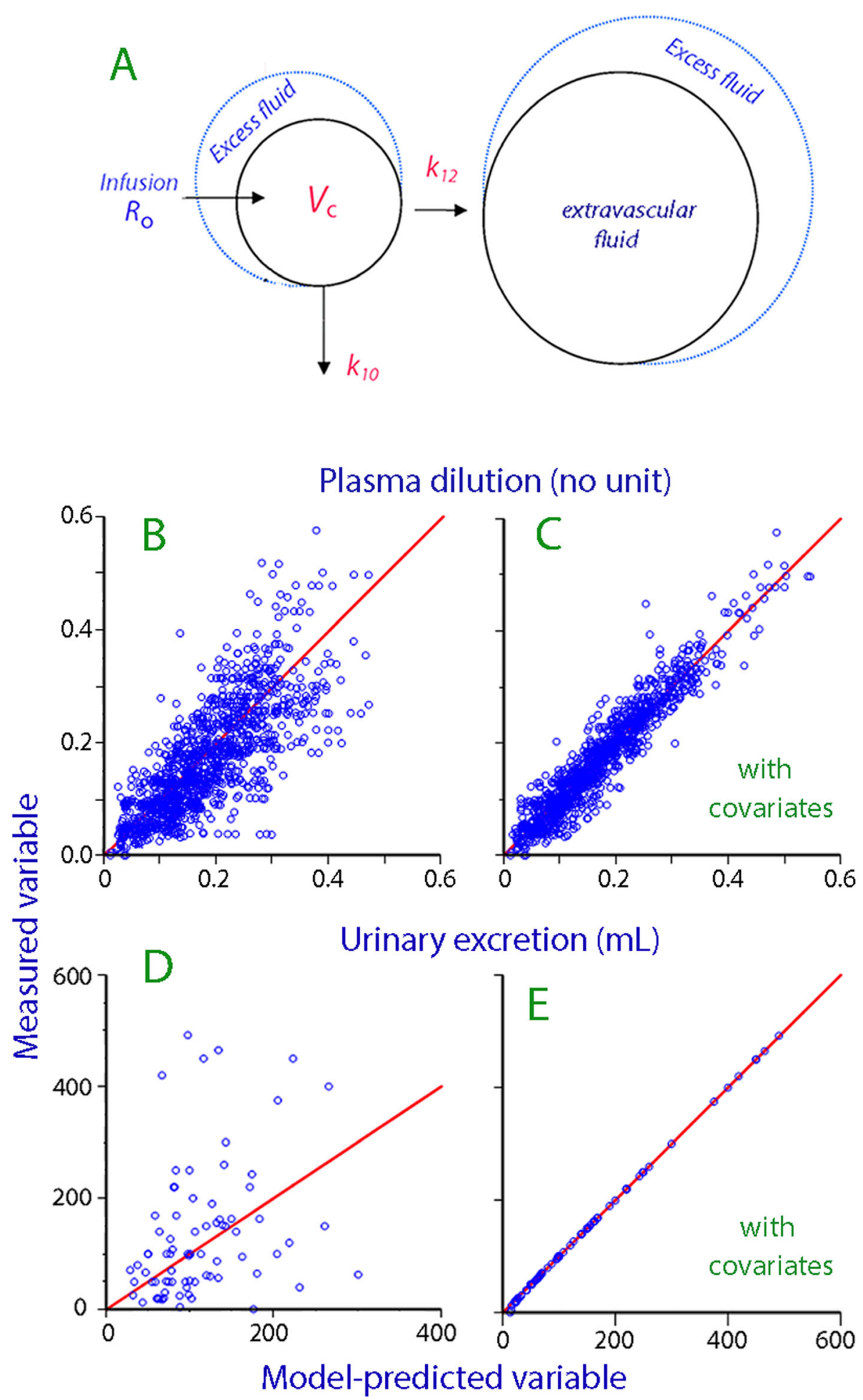

Fig. 1 Kinetic model and goodness-of-fit. (A) Schematic drawing of the volume kinetic model. (B) Predicted versus measured plasma dilution for all data points to the base model. (C) Same plot after correction for the covariates. Random distribution around the solid line of unity indicates a good goodness-of-fit. (D) Predicted versus measured urinary excretion for all patients according to the base model. (E) Same plot after correction for covariates 
with the First-Order Conditional Estimation Extended Least Squares as a search routine and an additive model for the random-error variability (Owen and Fiedler-Kelly 2014). While the covariates were added with a diagonal design, the finally reported parameter values represent a "Full Block Model" that considers correlations between random effects and is considered more accurate for simulation purposes.

The goodness-of-fit of the model was studied by residual plotting, where the dependent variables were recreated from the fixed parameters with and without consideration of the covariates.

The performance of the model was illustrated by predictive checks (1000 iterations) and bootstrap analysis (500 runs, with random sampling and replacement), using the built-in features of the Phoenix software.

Demographic data were reported as the mean (standard deviation), and the kinetic data were reported as the mean (95\% confidence interval).

\section{Results}

Table 1 shows the characteristics of the 76 infusion experiments. The patient mean age was 63 (standard deviation, 15) years, the mean body weight was 66 (17) kg, and each patient received 1157 (323) mL of Ringer's. The kinetic analysis was based on 1198 measurements of plasma dilution and 128 measurements of the urine volume. The original data are given in Supplementary file 1 .

\section{Base model}

The search for an optimal base model resulted in a number of modifications of the conventional twovolume model (Fig. 1A). The most important change is that the rate constant $k_{21}$ was removed due to lack of statistical significance. Several add-ons were tried that have occasionally been included in previous work. These include a second elimination function and an absorption function, but none of them improved the model. Hence, the variant described in the "Methods" section, with one expandable fluid space $\left(V_{\mathrm{c}}\right)$, one distribution $\left(k_{12}\right)$, and one elimination $\left(k_{10}\right)$ function, was found to be optimal. The search strategy used to find the final population kinetic model is shown in Table 2 .

\section{Included covariates}

The covariate search showed that the body weight (BW) affected $V_{\mathrm{c}}$ and that the absolute (crude) value of MAP significantly influenced both $k_{12}$ and $k_{10}$. The model parameters in the final analysis are shown in Table 3, which explains that the values of the fixed (group) parameters were modified by the body weight and by the individual-specific MAP at each timepoint to create the following unique model parameter for each timepoint of each individual patient:

$$
\begin{aligned}
& V_{c}=2,146\left[(\mathrm{BW} / 66)^{0.86}\right] \\
& k_{12}=22.1 \times 10^{-3}\left[(\mathrm{MAP} / 94.4)^{2.34}\right] \\
& k_{10}=5.1 \times 10^{-3}\left[(\mathrm{MAP} / 94.4)^{3.73}\right]
\end{aligned}
$$

where 66 is the mean patient body weight and 94.4 is the mean MAP for all 1198 observations. The MAP at baseline was 107.3 (standard deviation, 9.3) $\mathrm{mmHg}$.

\section{Discarded covariates}

Several potential covariates were tested that did not reach statistical significance. These included age; gender; ratio and change of MAP from baseline; the use of ephedrine $(n=13)$; use of regional/general anesthesia; and the time period before versus after the induction. Acetated Ringer's seemed to distribute more rapidly than lactated Ringer's, but this difference lost significance in the final model.

In accordance with a previous study (Hahn and Nemme 2020), the distribution $\left(k_{12}\right)$ was hypothesized to

\begin{tabular}{|c|c|c|c|c|c|}
\hline Optimization routine & Model & Target parameter & LL & $-2(\mathrm{LL})$ & AIC \\
\hline Naive pooled & 5 parameters $\left(V_{c}, k_{12}, k_{21}, k_{10}\right.$, and $\left.k_{b}\right)$ & & 340 & -681 & -631 \\
\hline Naive pooled & Removal of first fixed parameter & $k_{\mathrm{b}}$ & 341 & -681 & -669 \\
\hline FOCE ELS & Removal of first fixed parameter & $k_{\mathrm{b}}$ & 1280 & -2560 & -2540 \\
\hline FOCE ELS & Removal of second fixed parameter & $k_{21}$ & 1541 & -3081 & -3065 \\
\hline \multirow[t]{2}{*}{ FOCE ELS } & Add covariate: body weight & $V_{c}$ & 1558 & -3117 & -3099 \\
\hline & Add covariate: crude MAP & $k_{12}$ & 1605 & -3210 & -3190 \\
\hline FOCE ELS & Add covariate: crude MAP & $k_{10}$ & 1632 & -3264 & -3242 \\
\hline FOCE ELS & Full block model & All the above & 1652 & -3304 & -3276 \\
\hline FOCE ELS & Bootstrap analysis & All the above & 1652 & -3304 & -3246 \\
\hline
\end{tabular}

Table 2 Key features of the search protocol used to build the final population kinetic model. The strategy is to reduce $-2(\mathrm{LL})$ by more than 3.8 points in each step, which means that the change of the model is statistically significant 
Table 3 Population kinetic parameters in the final model

\begin{tabular}{|c|c|c|c|c|c|}
\hline & Covariate & Best estimate & $95 \% \mathrm{Cl}$ & RSE & $\begin{array}{l}\text { Bootstrap } \\
95 \% \mathrm{Cl}\end{array}$ \\
\hline \multicolumn{6}{|l|}{ Kinetic parameter } \\
\hline$V_{c}(L)$ & & 2.15 & $1.88-2.42$ & 6.4 & $1.88-2.45$ \\
\hline$k_{12}\left(10^{-3} \min ^{-1}\right)$ & & 22.1 & $15.3-28.8$ & 15.6 & $16.3-29.1$ \\
\hline$k_{10}\left(10^{-3} \min ^{-1}\right)$ & & 5.1 & $3.9-6.4$ & 12.1 & $4.0-6.5$ \\
\hline \multicolumn{6}{|l|}{ Covariate effects } \\
\hline$V_{c}$ & Body weight & 0.86 & $0.51-1.21$ & 19.8 & $0.51-1.16$ \\
\hline$k_{12}$ & MAP & 2.34 & $1.87-2.82$ & 10.4 & $1.65-3.36$ \\
\hline$k_{10}$ & MAP & 3.73 & $1.99-5.47$ & 23.7 & $2.31-5.74$ \\
\hline
\end{tabular}

$V_{c}$ central volume of distribution; conversion factor between plasma dilution and plasma volume expansion, $k_{12}$ rate constant for translocation of fluid from $V_{c}$ to the extravascular fluid space, $k_{10}$ rate constant governing urinary excretion, $C l$ confidence interval, $R S E$ standard error/best estimate

be turned off for a period of time after the induction; therefore, "turn-off" time periods of 3, 5-6, 9-10, 15, 20, and 25 min were tested. The amount of fluid infused up to those timepoints was evaluated, but none of those variables was strong enough for inclusion in the model.

\section{Illustrations}

Figure $1 \mathrm{~B}-\mathrm{E}$ shows the ability of the final model to recreate the dependent variables with and without consideration of the covariates. Comparison of Fig. 1D and E illustrates the great importance of MAP to the urinary excretion.
The distributions of the data on plasma dilution and MAP are given in Fig. $2 \mathrm{~A}-\mathrm{B}$, and their relationship is shown in Fig. 2C.

The influence of MAP on $k_{12}$ and $k_{10}$ is shown graphically in Fig. 2D.

The performance of the model in the form of a predictive check based on 1000 simulations is illustrated in Fig. 2E.

\section{Secondary calculations}

The model parameters in Table 3 were used to perform secondary calculations. These serve to illustrate the relevance of the kinetic data.
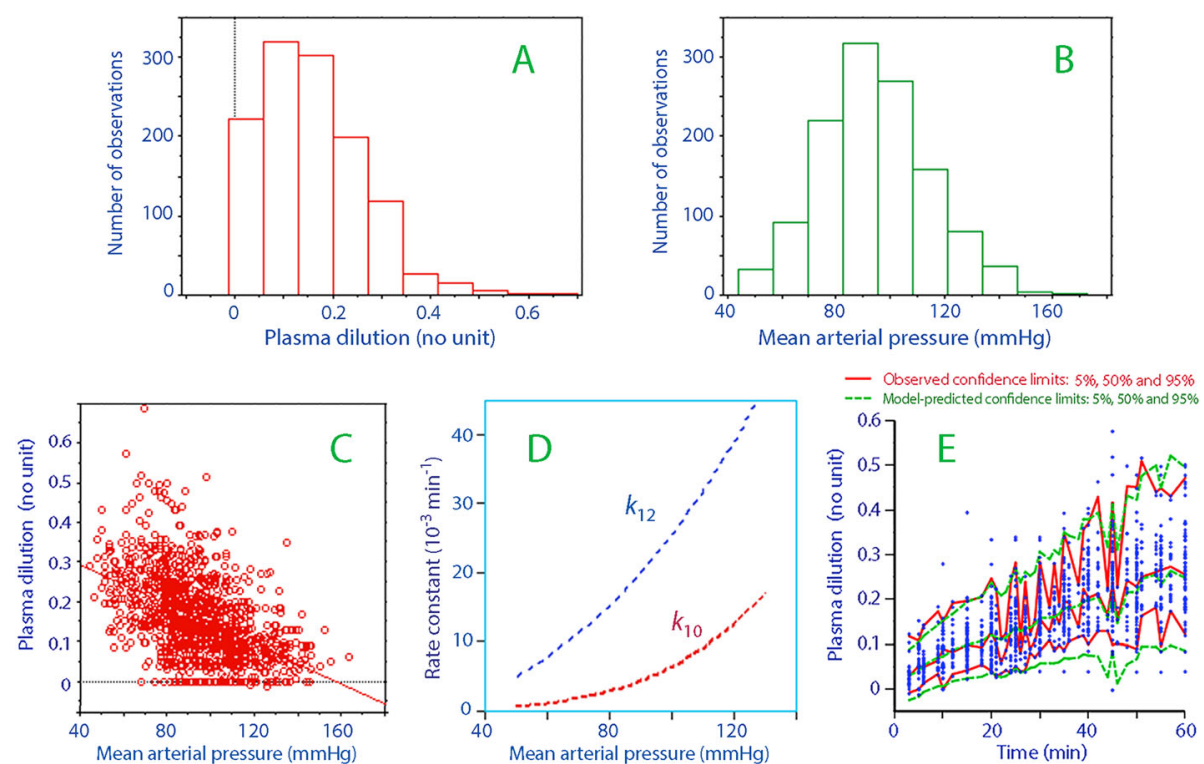

Fig. 2 Distribution of observations of plasma dilution $(\mathbf{A})$ and mean arterial pressures (B) and the relationship between these variables (C) throughout the study in all 76 patients, illustrating the limits for simulation by using the kinetic parameters. (D) Decrease in rate parameters for lower mean arterial pressures. (E) Predictive check showing the original data (blue points) with their confidence limits (red). The confidence limits based on 1000 simulations using the model parameters in the final model are superimposed (green lines). Hatched pattern is due to different sampling times. A small difference between observed and predicted confidence limits is a sign of good model performance. The difference between observed and predicted dilution averaged $0.03(0.02)$ at the $5 \%$ level, $-0.01(0.05)$ at the $50 \%$ level, and $-0.06(0.06)$ dilution units at the 95\% level 
Figure 3 shows the magnitude of the fluid retention occurring when MAP decreases by entering the model parameters into a simulation program.

The rate constants can be used to calculate the distribution and elimination half-lives for different MAP. For example, the intravascular half-life $\mathrm{T}_{1 / 2}=$ natural logarithm of $2(=0.693) /\left(k_{10}+k_{12}\right)$ was 15 min at a MAP of 110 $\mathrm{mmHg}$ while being $80 \mathrm{~min}$ at a MAP of $60 \mathrm{mmHg}$. The corresponding values for the elimination $\mathrm{T}_{1 / 2}$, obtained as $\ln 2 / k_{10}$, were $77 \mathrm{~min}$ and $737 \mathrm{~min}$, respectively.
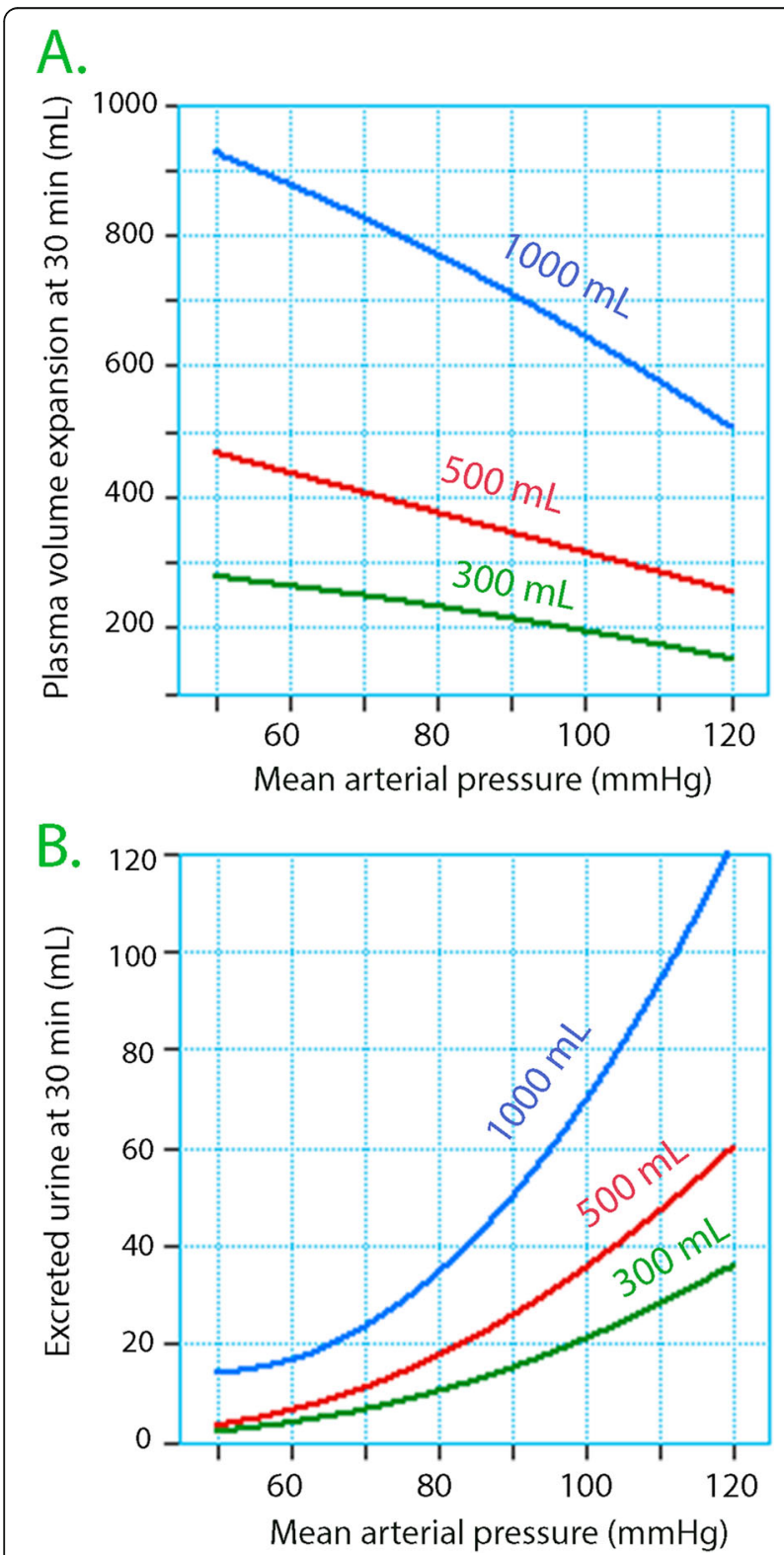

Fig. 3 Simulations. Computer simulation showing $(\mathbf{A})$ the plasma volume expansion and (B) the excreted urine volume at the end of a 30-min infusion of various volumes of crystalloid fluid. The parameter values from Table 3 were used

\section{Discussion}

\section{Main findings}

This report provides a mathematical description of what happens to the fluid distribution when anesthesia is induced in the middle of a continuous crystalloid infusion. The most apparent effect consists of a marked reduction in the rate constant that governs the distribution of infused fluid $\left(k_{12}\right)$ from the central fluid space $\left(V_{c}\right.$, the plasma) to the extravascular space. Fluid was distributed more slowly.

This change is probably an illustration of the classical Starling equation, which holds that the transcapillary exchange of fluid is determined by the balance between hydrostatic and oncotic forces across the capillary wall. A lowered MAP is likely to decrease the intravascular hydrostatic pressure. This, in turn, would reduce the capillary filtration because the interstitial hydrostatic pressure remains unchanged.

The elimination rate constant that describes urinary excretion $\left(k_{10}\right)$ also decreased, and to an even greater extent than was observed for the distribution. Both reductions were proportional in a non-linear fashion to the anesthesia-induced decrease in MAP, as shown in Fig. 2D and illustrated in Fig. 3.

\section{Clinical implications}

The excessive intravascular accumulation of infused fluid during induction of anesthesia is apparently a consequence of the vasodilatation which degree is indicated by the decrease in MAP. There is limited evidence to suggest that the volume expansion would affect the arterial pressure except if vary large volumes are infused and, in particular, if the crystalloid fluid is replaced by a colloid (James and Dyer 2016). However, an often overlooked issue is that the volume expansion is mirrored by a reduction of the blood $\mathrm{Hb}$ level that will be much greater expected as a result of the vasodilatation, and this is relevant for perioperative medicine. Marked $\mathrm{Hb}$ changes occur even without hemorrhage when MAP is modulated by regional or general anesthesia. For example, the drop in $\mathrm{Hb}$ will decrease oxygen delivery if it is unmatched by an increase in the stroke volume. Furthermore, a pre-set $\mathrm{Hb}$ used as transfusion trigger will be reached more rapidly than is indicated by the surgical blood loss.

A dependency of the plasma volume expansion on MAP was observed previously in the papers underlying this work (Ewaldsson and Hahn 2001, 2005; Li et al. 2007). However, the present population kinetic analysis of the pooled data provides a more precise understanding of this relationship. The data presented here even allow simulations to be made that predict how variations in MAP and the amount and rate of infused fluid affect $\mathrm{Hb}$. The following example, based on mass balance 
calculations (Ho et al., 2016), illustrates the influence of the reported fluid retention as compared to the conventional view of how fluid affects the blood $\mathrm{Hb}$ concentration. A widely cited relationship holds that infusion of 1 $\mathrm{L}$ of crystalloid increases the blood volume by $150 \mathrm{~mL}$ (Jacob et al., 2012), which would reduce blood $\mathrm{Hb}$ from $150 \mathrm{~g} / \mathrm{L}$ to $144 \mathrm{~g} / \mathrm{L}$ if the baseline blood volume is $5 \mathrm{~L}$. Based on the relationships presented here, infusion of the same amount over 30 min during the induction of anesthesia would reduce $\mathrm{Hb}$ to $129 \mathrm{~g} / \mathrm{L}$ if the MAP is 70 $\mathrm{mmHg}$; i.e., 3.5 times more.

Some of this difference is not due to MAP but to the fact that crystalloid fluid shows a distribution function that requires 25-30 min for completion. In the example above, as much as $83 \%$ of the infused volume remains in the blood, and the patient will be close to being anuric if the anesthesia reduces MAP to $70 \mathrm{mmHg}$ (Fig. 3). However, induction of general or regional anesthesia with unchanged MAP was still associated with a plasma volume expansion amounting to $50 \%$ at the end of a 30 min infusion of Ringer's. This confirms previous findings in volunteers (Hahn 2010) and is 3 times greater than after the infusion is completed (Jacob et al. 2012).

The increased plasma volume expansion due to the MAP-dependent decrease in $k_{12}$ is likely to remain until the intravascular hydrostatic pressure has increased sufficiently to reach a new Starling equilibrium, and this increase requires a vasoconstrictor, capillary refill, or additional infusion of fluid. The $k_{10}$ value is known to remain low, despite adequate volume compensation, as long as MAP is low, but the normal value is resumed when the patient awakens from the anesthesia (Hahn 2020).

\section{Kinetic analysis}

Several variables were evaluated that did not receive sufficient strength to be included in the kinetic model. For example, ephedrine administration had only an indirect effect via MAP on the kinetic parameters. Previous work has shown that buffered Ringer's solution undergoes a more rapid turnover in young subjects than in aged subjects, but the current age span was probably too narrow to distinguish that relationship. No differences in fluid kinetics were found between patients who received spinal, epidural, or general anesthesia. The crude MAP, and not the change from baseline, governed the fluid kinetics, just as occurs during ongoing surgery (Hahn 2017).

A thorough evaluation was made to determine whether a normal $k_{12}$ was resumed after a certain amount of fluid had been infused. However, no such "turning point" was found. The reason is probably that the infusion of $1.1 \mathrm{~L}$ of Ringer's did not fill up the vasodilated cardiovascular system sufficiently to allow a resumption of the normal exchange of fluid with the extravascular space. In a previous study, this "turning point" was reached when $16.6 \mathrm{~mL} / \mathrm{kg}$ of Ringer's (1.25 L) had been administered (Hahn and Nemme 2020). This probably corresponds to the anesthesia-induced expansion of the part of the blood volume that is sometimes called "unstressed" and which denotes the amount of venous blood that does not increase the transmural pressure (Gelman 2008).

\section{Current versus previous models}

The excessive accumulation of infused Ringer's solution during the onset of regional anesthesia was first studied by linear regression and reported in the early 1990s (Hahn 1992). A later study showed that the decrease in MAP appears a few minutes before the increased hemodilution, and this finding clarified the order of events (Drobin and Hahn 1996). Subsequent analyses of the fluid kinetics during induction of anesthesia applied a clearance version with a single inter-compartmental clearance parameter (Li et al. 2007), but this is problematic because the Starling forces are changed in the middle of the experiment. The present population kinetic model separates the flows in and out of the plasma volume and shows clearly that no return of fluid to the plasma occurs during the onset of anesthesia as long as fluid loading is ongoing.

The current model also uses micro-constants instead of clearances, which makes it independent of plasma volume and body fluid volumes. The micro-constant model detects a "wall" between a central space, where fluid equilibrates very rapidly with the site of infusion, and a more remote peripheral space. The space with this fast equilibration is very likely to represent the plasma volume contained in blood vessels that are allowed to expand. The exchange of infused fluid between these two body fluid spaces is determined by rate constants $\left(k_{12}\right.$ and $k_{21}$ ). The volume of the infused fluid residing in the two body fluid spaces is obtained directly in the microconstant model, while their dilution must be multiplied by the volume of distribution to obtain volume expansion in the clearance model.

\section{Limitations}

The limitations of the present study include that the data were obtained from three previously published works, although all three used a similar protocol and sampling had been performed in the same way.

The fairly low values of $k_{12}$ and $k_{10}$ before the induction may reflect preoperative anxiety, which has been observed in adults and children alike (Li et al. 2009). Higher values would be expected if the fluid had been infused in volunteers not scheduled for surgery (Hahn 2020).

The strength of this study is that a modern and robust kinetic approach was used that allows simultaneous analysis of all studied patients, as well as comparisons of 
several different covariances that could potentially influence the fluid kinetics.

\section{Conclusion}

The induction of regional and general anesthesia was followed by a MAP-dependent decrease in both the distribution and elimination of an infused crystalloid fluid. Both changes markedly increased the hemodilution, which might become large enough to affect oxygen delivery and distort schemes for deliberate hemodilution and estimates of allowable blood loss.

\begin{abstract}
Abbreviations
$H b$ : Hemoglobin; i.v.: Intravenous; $k_{12}$ : Rate constant for fluid passing from $v_{c}$ to the extravascular fluid space; $k_{21}$ : Rate constant for fluid passing from the extravascular space to $v_{c} ; k_{10}$ : Rate constant for fluid eliminated by urinary excretion; $k_{\mathrm{b}}$ : Rate constant for eliminated fluid not recovered as urine; MAP: Mean arterial pressure; NLME: Non-linear mixed effects; $R_{0}$ : Infusion rate; $V_{c}, v_{c}:$ Sizes of central body fluid space at baseline and during fluid therapy, respectively; $T_{1 / 2}$ : Half-life
\end{abstract}

\section{Supplementary Information}

The online version contains supplementary material available at https://doi. org/10.1186/s13741-021-00204-5.

\section{Additional file 1}

\section{Acknowledgements}

The author is grateful to the coauthors and others who contributed to the original papers on which the present work is based.

\section{Author's contributions}

RGH planned all three studies. RGH planned the present report, made the calculations, and wrote the manuscript. The author read and approved the final manuscript.

\section{Funding}

Departmental funds. Open Access funding provided by Karolinska Institute.

\section{Availability of data and materials}

The data used for the kinetic analysis are available as Supplementary file 1.

\section{Declarations}

\section{Ethics approval and consent to participate}

These studies were conducted at Huddinge University Hospital, Sweden, Dnr. 350/00, Karolinska Institutet, Sweden, 97/123, and First Affiliated Hospital in Hangzhou, Zhejiang University, PR of China, Dnr. 050156. The studies were initiated before database registrations of clinical trials became mandatory. No new application for ethics approval for the present pooled analysis was made because informed consent had already been obtained in the included trials.

\section{Consent for publication}

Not applicable.

\section{Competing interests}

RGH holds a grant from Grifols for the study of $20 \%$ albumin as infusion fluid.

Received: 3 May 2021 Accepted: 25 June 2021

Published online: 11 October 2021

\section{References}

Coe AJ, Revenäs B. Is crystalloid preloading useful in spinal anaesthesia in the elderly? Anaesthesia. 1990:45(3):241-3. https://doi.org/10.1111/j.1365-2044.1 990.tb14696.x.
Drobin D, Hahn RG. Time course of increased haemodilution in hypotension induced by extradural anaesthesia. Br J Anaesth. 1996;77(2):223-36. https:/ doi.org/10.1093/bja/77.2.223.

Ewaldsson CA, Hahn RG. Bolus injection of Ringer's solution and dextran 1 kD during induction of spinal anesthesia. Acta Anaesthesiol Scand. 2005;49(2): 152-9. https://doi.org/10.1111/j.1399-6576.2004.00561.x.

Ewaldsson C-A, Hahn RG. Volume kinetics during induction of spinal and general anaesthesia. Br J Anaesth. 2001;87(3):406-14. https://doi.org/10.1093/bja/ 87.3.406.

Gelman S. Venous function and central venous pressure. Anesthesiology. 2008; 108(4):735-48. https://doi.org/10.1097/ALN.0b013e3181672607.

Hahn RG. Haemoglobin dilution from epidural-induced hypotension with and without fluid loading. Acta Anaesthesiol Scand. 1992;36(3):241-4. https://doi. org/10.1111/j.1399-6576.1992.tb03457.x.

Hahn RG. Volume kinetics for infusion fluids. Anesthesiology. 2010;113(2):470-81. https://doi.org/10.1097/ALN.0b013e3181dcd88f

Hahn RG. Arterial pressure and the rate of elimination of crystalloid fluid. Anesth Analg. 2017;124(6):1824-33. https://doi.org/10.1213/ANE.0000000000002075.

Hahn RG. Understanding volume kinetics. Acta Anaesthesiol Scand. 2020;64(5): 570-8. https://doi.org/10.1111/aas.13533.

Hahn RG, Nemme J. Volume kinetic analysis of fluid retention after induction of general anaesthesia. BMC Anaesthesiol. 2020;20(1):95. https://doi.org/10.1186/ s12871-020-01001-1.

Heeremans EH, Proost JH, Eleveld DJ, Absalom AR, Struys MRF. Population pharmacokinetics and pharmacodynamics in anesthesia, intensive care and pain medicine. Curr Opin Anesthesiol. 2010;23(4):479-84. https://doi.org/10.1 097/ACO.0b013e32833a1d2f.

Ho L, Lau L, Churilov L, Riedel B, McNicol L, Hahn RG, et al. Comparative evaluation of crystalloid resuscitation rate in a human model of compensated haemorrhagic shock. Shock. 2016;46:147-57.

Jacob M, Chappell D, Hofmann-Kiefer K, Helfen T, Schuelke A, Jacob B, et al. The intravascular volume effect of Ringer's lactate is below 20\%: a prospective study in humans. Crit Care. 2012;16(3):R86. https://doi.org/10.1186/cc11344.

James MFM, Dyer RA. Spinal anesthesia. In: Hahn RG, editor. Clinical fluid therapy in the perioperative setting. 2nd ed. Cambridge: Cambridge University Press; 2016. p. 141-8.

Li Y, Hahn RG, Hu Y, Xiang Y, Zhu S. Plasma and renal clearances of lactated Ringer's solution in pediatric and adult patients just before anesthesia is induced. Pediatr Anesth. 2009;19(7):682-7. https://doi.org/10.1111/j.14609592.2009.03047.x.

Li Y, Zhu S, Hahn RG. The kinetics of Ringer's solution in young and elderly patients during induction of general anesthesia with propofol and epidural anesthesia with bupivacaine. Acta Anaesthesiol Scand. 2007;51:880-7.

McCrae AF, Wildsmith JAW. Prevention and treatment of hypotension during central neural block. Br J Anaesth. 1993;70(6):672-80. https://doi.org/10.1093/ bja/70.6.672.

Owen JS, Fiedler-Kelly J. Introduction to population pharmacokinetic/ pharmacodynamic analysis with nonlinear mixed effects models. Hoboken: Wiley; 2014. https://doi.org/10.1002/9781118784860.

\section{Publisher's Note}

Springer Nature remains neutral with regard to jurisdictional claims in published maps and institutional affiliations.

Ready to submit your research? Choose BMC and benefit from:

- fast, convenient online submission

- thorough peer review by experienced researchers in your field

- rapid publication on acceptance

- support for research data, including large and complex data types

- gold Open Access which fosters wider collaboration and increased citations

- maximum visibility for your research: over $100 \mathrm{M}$ website views per year

At BMC, research is always in progress.

Learn more biomedcentral.com/submissions 